\title{
Recurrent Malignant Bone Neoplasm
}

National Cancer Institute

\section{Source}

National Cancer Institute. Recurrent Malignant Bone Neoplasm. NCI Thesaurus. Code C150524.

Reemergence of a malignant bone neoplasm after a period of remission. 